\title{
Surgical anatomy of the sciatic nerve and its relationship to the piriformis muscle with a description of a rare variant
}

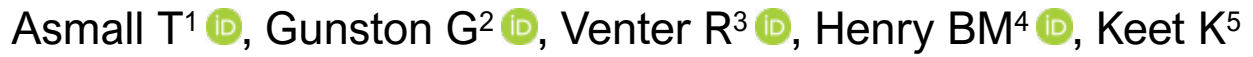

1 BSc(Hons); Post-graduate student, Division of Clinical Anatomy and Biological Anthropology, Department of Human Biology, Faculty of Health Sciences, University of Cape Town, South Africa

2 MBChB, MPhil Ed; Senior lecturer, Division of Clinical Anatomy and Biological Anthropology, Department of Human Biology, Faculty of Health Sciences, University of Cape Town, South Africa

3 MBChB, FC Orth, MMed; Orthopaedic surgeon, Division of Clinical Anatomy, Department of Biomedical Sciences, Faculty of Medicine and Health Sciences, Stellenbosch University, South Africa and Division of Orthopaedic Surgery, Department of Surgical Sciences, Tygerberg Hospital, South Africa

4 MD; Coordinator, The Heart Institute, Cincinnati Children's Hospital Medical Center, Cincinnati, Ohio, United States of America

5 MSc; Lecturer, Division of Clinical Anatomy, Department of Biomedical Sciences, Faculty of Medicine and Health Sciences, Stellenbosch University, South Africa

Corresponding author: Ms Kerri Keet, F103, Fisan Building, Faculty of Medicine and Health Sciences, Francie van Zyl Drive, Tygerberg Hospital, Cape Town, South Africa, 7505; tel: +27279389754; email: keetkerri@gmail.com

\section{Abstract}

Aims: Variation of the sciatic nerve may increase the risk of iatrogenic injury during total hip arthroplasty or arthroscopy, result in failure of peripheral blocks, or be associated with piriformis syndrome. Studies from Africa are scarce, with none to date from South Africa. Thus, the aims were to document the relationship between the sciatic nerve and piriformis muscle, variation in the bifurcation level and in the length of the nerve. Any significant differences between sexes and sides were also investigated.

Methods: The lower limbs of 42 cadavers (84 limbs) were dissected and the relationship between the sciatic nerve and piriformis classified according to the patterns described by Beaton and Anson. The region of sciatic nerve bifurcation was documented, and the length of the nerve was measured in individuals with bifurcation in the thigh.

Results: The normal relationship between the sciatic nerve and piriformis muscle was present in 64 limbs (76.2\%). The bifurcation level of the nerve was variable in more than half the sample. No significant differences occurred in any of the variant patterns or bifurcation regions between side or sex; however, variations were more common in females than in males. The mean length of the sciatic nerve was $133.30 \pm 19.33 \mathrm{~mm}$, with no differences in length between sex or side.

Conclusion: Variations in the anatomy of the sciatic nerve occurred in up to half of the sample, which may have implications for increased risk of iatrogenic injury in total hip arthroplasty and arthroscopy, piriformis syndrome or sciatic block failure.

Level of evidence: Level 4

Keywords: anatomical variation, piriformis muscle, piriformis syndrome, sciatic nerve, total hip arthroplasty

Citation: Asmall T, Gunston G, Venter R, Henry BM, Keet K. Surgical anatomy of the sciatic nerve and its relationship to the piriformis muscle with a description of a rare variant. SA Orthop J 2020;19(1):33-39. http://dx.doi.org/10.17159/2309-8309/2020/v19n1a5

Editor: Dr C Snyckers, University of Pretoria, Pretoria, South Africa

Received: May 2019

Accepted: September 2019

Published: March 2020

Copyright: ( 2020 Asmall T, et al. This is an open-access article distributed under the terms of the Creative Commons Attribution Licence, which permits unrestricted use, distribution and reproduction in any medium, provided the original author and source are credited.

Funding: This work was supported by the University of Cape Town and Royal Rice, who were not involved with the study design or outcomes.

Conflict of interest: The authors have no conflicts of interest to declare. 


\section{Introduction}

The sciatic nerve (SN) is formed in the pelvis from the ventral roots L4-S3 of the lumbosacral plexus. ${ }^{1}$ The nerve emerges from the greater sciatic foramen, and enters the gluteal region, inferior to the piriformis muscle (PM). It courses over the superior gemellus, tendon of obturator internus, inferior gemellus and quadratus femoris muscles and descends to the ischial tuberosity, passing deep to the gluteus maximus and the long head of the biceps femoris. ${ }^{2}$ The nerve then enters the posterior thigh and usually divides here into the tibial nerve (TN) and common fibular nerve (CFN). Division typically occurs in the lower third of the thigh, near the apex of the popliteal fossa. ${ }^{3}$ However, this bifurcation level is variable. ${ }^{4}$ Although it passes through the gluteal region, the SN does not give off any branches here. Instead it continues inferiorly to supply motor and sensory innervation to the posterior compartment of the thigh and all compartments of the leg (including all lower limb joints) via its branches. ${ }^{1,5}$

In 1937, Beaton and Anson were the first to categorise variations in the relationship between the $\mathrm{SN}$ and $\mathrm{PM}$ (types $A-G$ ). ${ }^{6}$ The normal type, type $\mathrm{A}$, is where the $\mathrm{SN}$ emerges as a single trunk below the PM. ${ }^{7}$ The pooled prevalence of type $\mathrm{A}$ has been reported as $85.2 \% .^{1}$

The course of the $\mathrm{SN}$ is of clinical importance in the surgical treatment of hip disorders using arthroscopy or open surgical approaches. Posterior surgical approaches to the hip include the Moore or Southern approach, used mostly for hip arthroplasty procedures, and the Kocher-Langenbeck approach, typically used to visualise the posterior acetabulum. 8,9

In arthroscopy, the joint is visualised with an arthroscope and instruments are introduced from one of several portals or standardised points of access to avoid critical structures. ${ }^{10}$ One of these portals, the postero-lateral portal, lies $2 \mathrm{~cm}$ posterior to the tip of the greater trochanter and has been associated with occasional injury to the $\mathrm{SN}$, especially if the limb is positioned in external rotation. ${ }^{11}$

An abnormal course of the $\mathrm{SN}$ in relation to the PM may cause entrapment and compression, resulting in piriformis syndrome (PS), an extra-spinal cause of sciatic nerve pain. ${ }^{12}$ High bifurcation of the $\mathrm{SN}$ in the gluteal or thigh regions may result in inadequate analgesia during popliteal blocks as only one of its branches may be successfully anaesthetised. ${ }^{13}$ Variations in the formation and bifurcation level of the $\mathrm{SN}$ indicate that a range of lengths are present in the population.

A meta-analysis by Tomaszewski et al. identified differences in the prevalence of variation between studies from different geographic regions, with a significant lack of studies from Africa, potentially resulting in an increased risk of sciatic nerve pathologies (sciatica) and preventable iatrogenic nerve injuries. ${ }^{1}$ As such, the aim of this study was to determine a) the prevalence of the relationship types present between the SN and PM, b) the bifurcation level of the SN, and c) the length of the SN in a South African sample. Any differences in the outcomes between side and sex were also investigated.

\section{Materials and methods}

\section{Study design}

A cross-sectional observational study of 43 embalmed, formalinfixed adult cadavers was conducted at our institution between April and November 2017. The sample included both lower limbs from all cadavers that were dissected by second-year medical students in that year. Exclusion criteria included cadavers in whom the relationship between the SN and PM could not be determined due to previous dissection, trauma or pathology.

\section{Dissection}

The gluteal, posterior thigh and popliteal regions were dissected for both the left and right lower limbs of each cadaver. The skin, fat and connective tissues were removed using a standard dissection kit and technique. The body was placed in the prone position and a midline skin incision was made with a scalpel (size 10 blade), extending from the sacrum to the coccyx, progressing laterally beneath the fold of each buttock. The skin of the thigh and popliteal fossa were also removed by means of a vertical midline incision from the fold of each buttock to the popliteal fossa. The skin was reflected to either side and the fat and fascia were removed. Next, the gluteus maximus was exposed and reflected laterally by cutting the muscle at the point of its origin from the iliac crest, posterolateral aspect of the sacrum and the sacrotuberous ligament. The gluteus medius was exposed and reflected inferiorly by means of an incision through its origin, on the lateral surface of the ilium. Gluteus minimus, the PM and the SN were cleared of fascia and exposed. The course of the SN was followed between the hamstring muscles of the posterior thigh and the popliteal fossa, until its bifurcation into the CFN and TN.

\section{Data collection}

The primary outcomes investigated in this study were a) the relationship types between the $\mathrm{SN}$ and $\mathrm{PM}, \mathrm{b})$ the region of bifurcation of the SN, and c) the mean length of the SN. Secondary outcomes included determining any significant differences in the primary outcomes between males and females, and between left and right sides.

The relationship between the SN and PM in each lower limb was classified into types described by Beaton and Anson ${ }^{6}$ (1937) and updated by Tomaszewski et al. ${ }^{1}$ (2016).

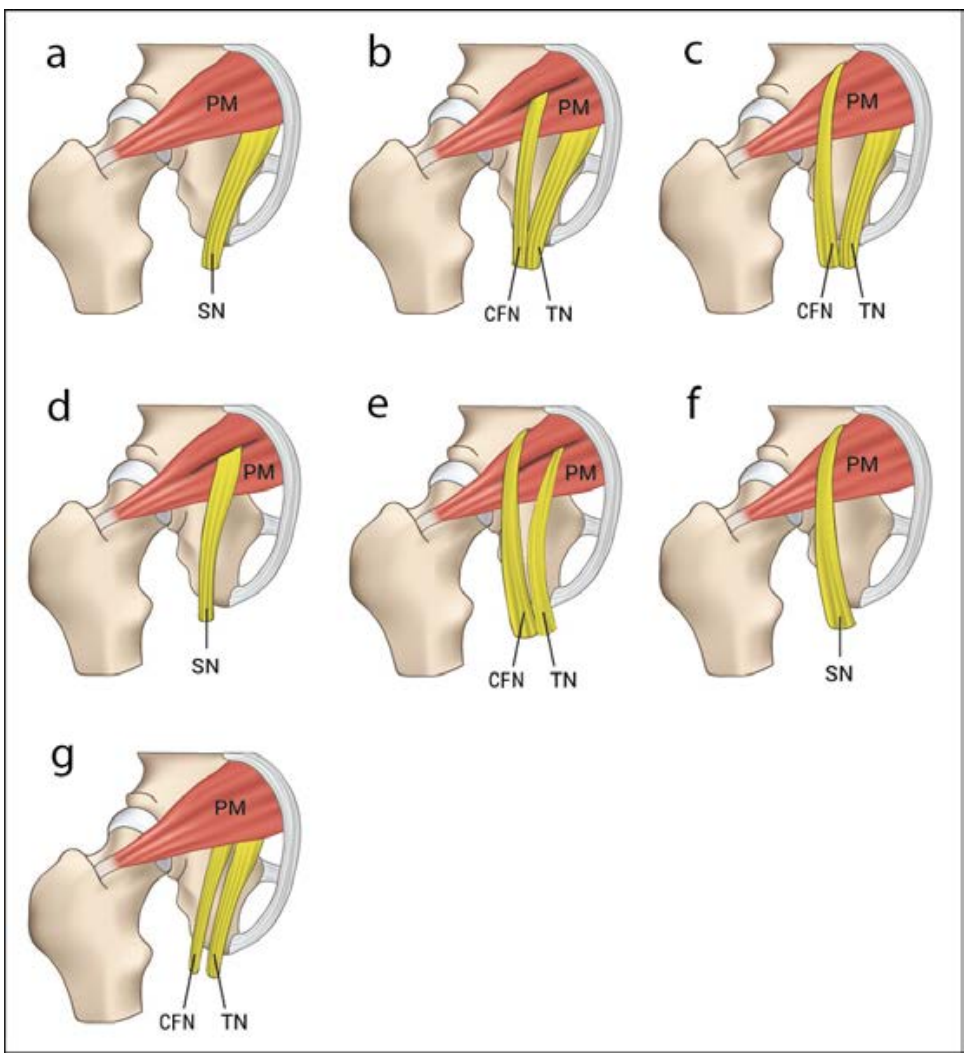

Figure 1. The different types $(a-g)$ of relationships between the sciatic nerve and piriformis muscle (figure reproduced with permission from Tomaszewski et al. ${ }^{1}$ ) $\mathrm{SN}$ : sciatic nerve; PM: piriformis muscle; TN: tibial nerve; CFN: common fibular nerve 
The description of each type of relationship between the $\mathrm{SN}$ and PM is as follows:

Type A: The SN passes undivided below the PM (normal relationship) (Figure 1a).

Type B: The SN divides in the pelvis, with the CFN piercing the PM and the TN emerging below the muscle (Figure $1 b$ ).

Type C: The SN divides in the pelvis, with the CFN emerging superior to the PM and the TN emerging below the muscle (Figure 1c).

Type D: The SN passes undivided through the belly of the PM (Figure 1d).

Type E: The SN divides in the pelvis, with the CFN emerging superior to the PM and the TN piercing the muscle (Figure 1e).

Type F: The SN emerges undivided superior to the PM and courses over the muscle (Figure 1f).

Type G: The SN divides in the pelvis, with both the CFN and TN emerging below the PM (Figure 1g).

The types were recorded for both the left and right legs of each individual in a tabular format in Microsoft Excel, and photographs were taken with a Huawei P8 Smartphone (13 megapixels). Two independent reviewers (anatomists) confirmed the pattern type for each limb to reduce the risk of observer bias.

Next, the region in which the SN bifurcated into the CFN and TN was documented. Division of the nerve in the area between the sacrum and the superior border of the quadratus femoris muscle was classified as a gluteal division; bifurcation between the inferior border of quadratus femoris and the apex of the popliteal fossa a thigh division; and division within the popliteal fossa was classified as a popliteal fossa division. The regions of bifurcation were confirmed by a second observer. For cadavers in which division occurred in the thigh, the $\mathrm{SN}$ was measured in situ from the midpoint of the inferior border of the quadratus femoris muscle to its bifurcation point. The inferior border of quadratus femoris was used as a reference point as it is a consistent and easily identified landmark in all individuals. Standard measuring equipment (an Edo shatter-resistant ruler, yarn and pins) were utilised and the measurements were recorded in millimetres in Microsoft Excel. Each measurement was taken three times, and the average of the three was used in the statistical analysis. Intra-observer bias was assessed by the principal researcher repeating the measurements after a period of one month. These measurements were then compared with the first set of measurements. Two independent reviewers, who were blinded to the study outcomes, measured the length of the SN in the assessment of inter-observer bias.

Information about height of the cadavers or limb length was not available, thus we were unable to correct for differences in length of the $\mathrm{SN}$ resulting from height variations.

\section{Statistical analysis}

All statistical analyses were performed in IBM SPSS ${ }^{\circledR}$ Version 24.0 (Armonk, New York, United States). Categorical data was represented as number $(\mathrm{n})$ and percentage $(\%)$. For numerical data, summary statistics for variables were reported as mean \pm standard deviation (SD) for normally distributed data, or median and interquartile range for non-normally distributed data.

Normal distribution of the numerical data was determined with the Shapiro-Wilk test. Intra- and inter-observer variability of numerical data was assessed using Bland-Altman plots and ANOVA tests. Significant differences in the prevalence of pattern types and bifurcation level were investigated between right and left sides and between males and females using chi-square or Fisher's exact tests. Differences in the length of the SN between the sex and side were investigated by means of unpaired Student's t-tests. For all statistical analyses, a p-value $<0.05$ was considered significant.

\section{Results}

\section{Subject characteristics}

Initially, 43 embalmed cadavers were included in the study. However, one male cadaver was excluded as the relationship between the $\mathrm{SN}$ and PM could not be determined due to previous dissection. This resulted in a final sample size of 42 cadavers (84 lower limbs), with $24(57.1 \%)$ males and 18 (42.9\%) females between the ages of 22 and 99 years. The cause of death in all individuals was natural.

\section{Prevalence of the types of relationships present between the SN and PM}

Type A was the most common pattern in $76.2 \%$, followed by type $G$ in $15.5 \%$ and lastly, type $B$ in $7.1 \%$ (Figures $2-4$ ). The other types (C, D, E, F) were not observed. An unclassified pattern was observed in one right lower limb (1.2\%) of a female cadaver, in which the SN emerged as two separate branches, with the CFN passing beneath the PM, while the TN coursed beneath the inferior gemellus muscle (Figure 5). There were no significant differences in prevalence of types between sides $(p=0.50)$ and sex $(p=0.08)$. The above-mentioned data is summarised in Table $l$.

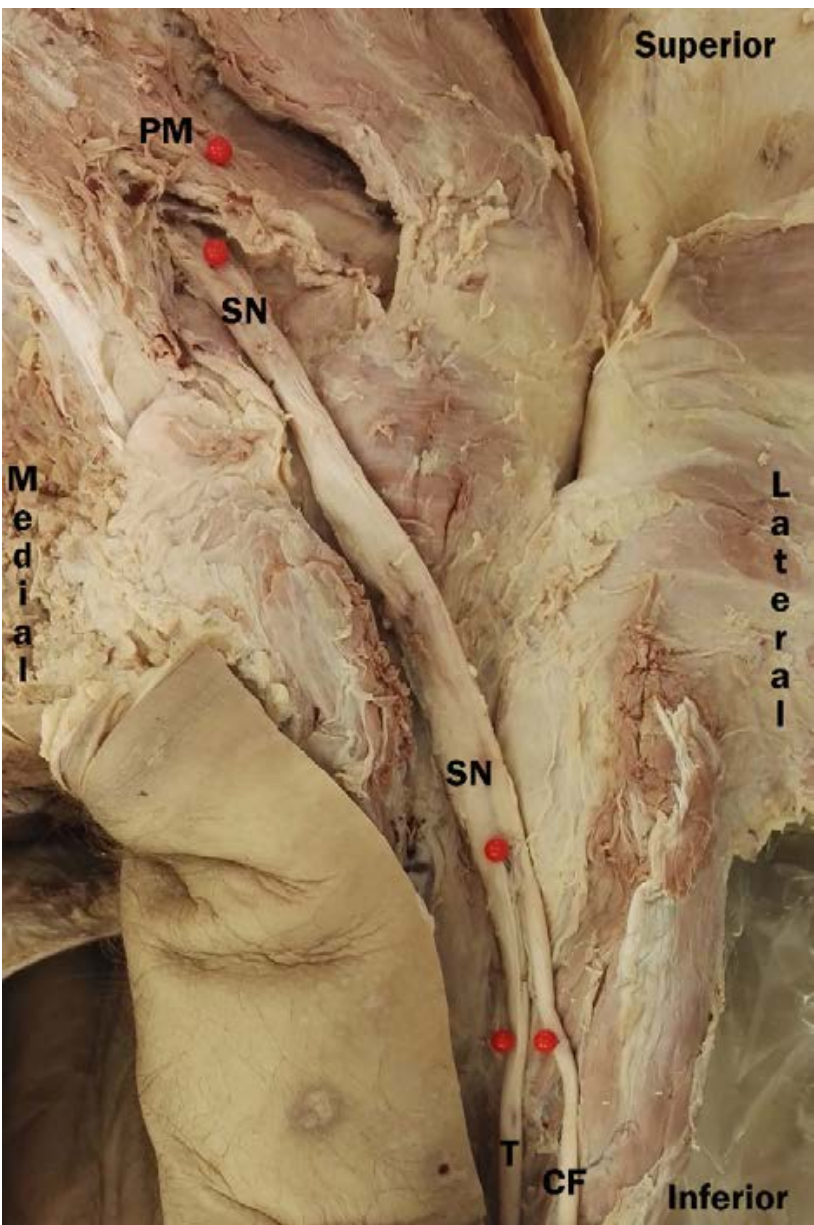

Figure 2. Type $A$ in which the sciatic nerve (SN) emerged undivided below the piriformis muscle (PM). In this individual, the sciatic nerve divided into tibial $(\mathrm{T})$ and common fibular (CF) nerves in the thigh. 


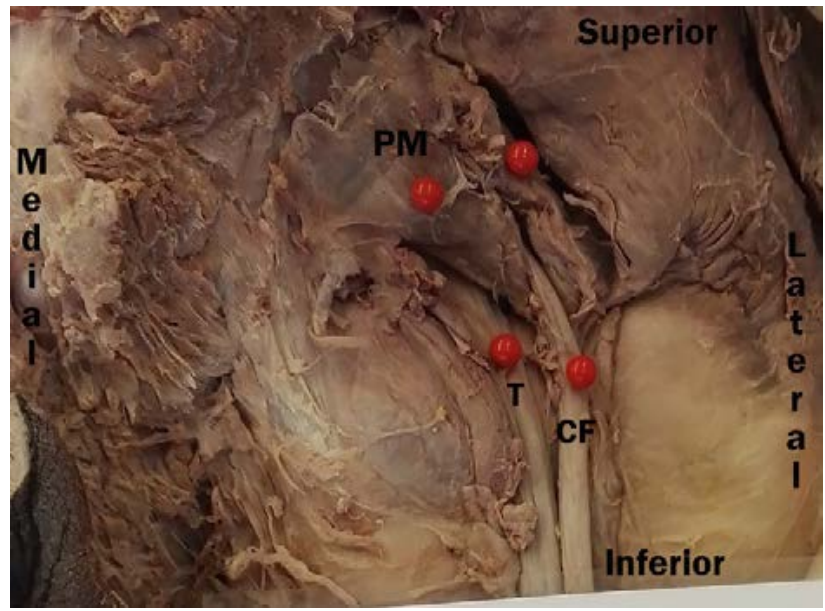

Figure 3. Type $B$ in which the sciatic nerve emerged as two separate branches, with the common fibular nerve (CF) piercing the piriformis muscle (PM), while the tibial nerve $(\mathrm{T})$ emerged below the muscle

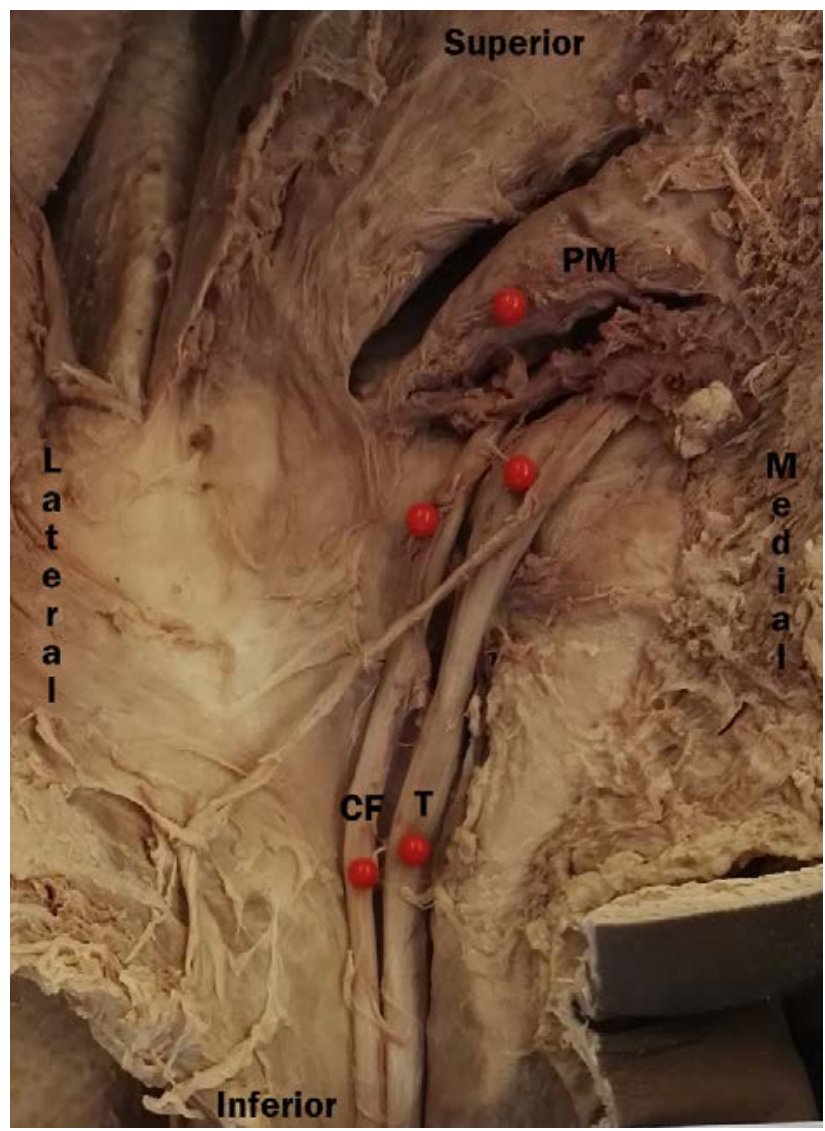

Figure 4. Type $G$ in which the tibial nerve $(T)$ and common fibular nerve (CF) emerged separately below the piriformis muscle (PM)

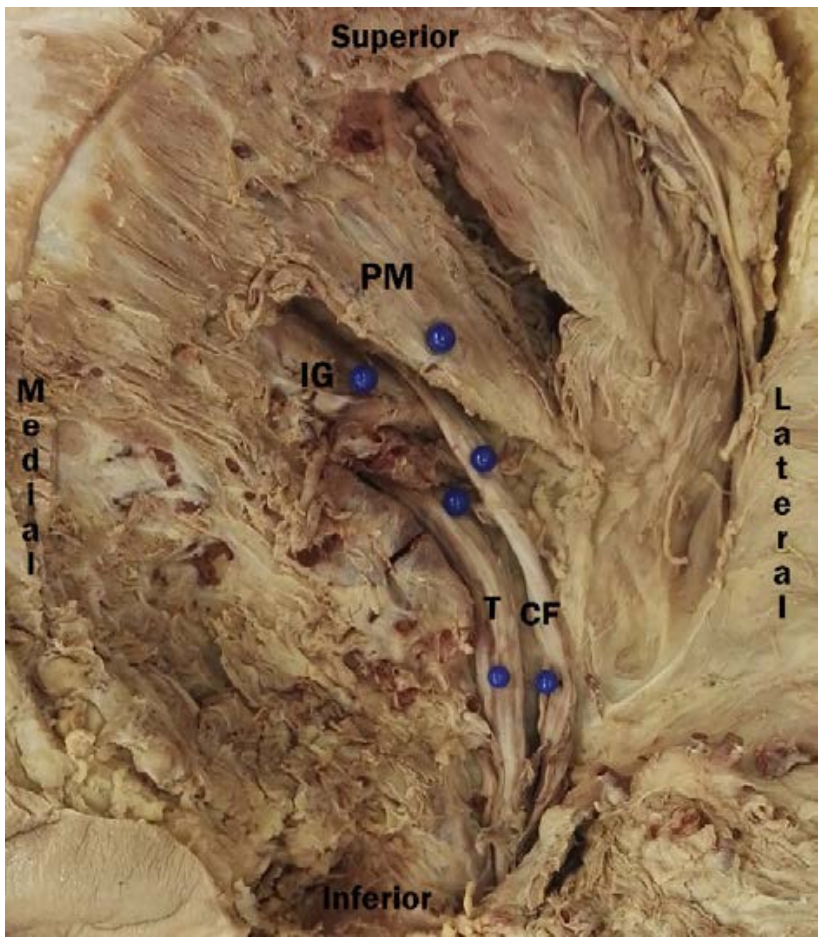

Figure 5. Unclassified type in which the common fibular nerve (CF) emerged below the piriformis muscle (PM), and the tibial nerve (T) coursed below the inferior gemellus (IG)

Of the 42 cadavers studied, 31 (73.8\%) showed symmetry in the pattern types. Type A was the most common pattern observed bilaterally (Table II). No significant differences were observed between males and females $(p=0.927)$ with respect to symmetry of types.

\section{Region of bifurcation of the sciatic nerve}

The sciatic nerve bifurcated in the gluteal, thigh and popliteal regions (Table III). Significant differences were observed between males and females $(p=0.02)$ in the region of $S N$ bifurcation. Bifurcation in the gluteal or thigh regions was $27.8 \%$ higher in males than in females, while bifurcation in the popliteal region was $27.7 \%$ more common in females than in males (Table III). No significant differences were observed between left and right sides $(p=0.73)$.

Of the 42 cadavers, $30(71.4 \%)$ displayed symmetrical division, with unilateral division present in 12 (28.6\%).

\section{Length of the sciatic nerve}

The length of the SN was measured for limbs in which the SN bifurcated in the thigh region. Two limbs were excluded as the SN

Table l: The prevalence of sciatic nerve variation types with respect to sex and side

\begin{tabular}{|l|c|c|c|c|c|}
\hline & $\begin{array}{c}\text { Total } \\
\mathbf{n}(\%)\end{array}$ & $\begin{array}{c}\text { Males } \\
\mathbf{n}(\%)\end{array}$ & $\begin{array}{c}\text { Females } \\
\mathbf{n}(\%)\end{array}$ & $\begin{array}{c}\text { Right } \\
\mathbf{n}(\%)\end{array}$ \\
\hline Type A & $64(76.2)$ & $35(73)$ & $29(81)$ & $32(76.2)$ & $32(76.2)$ \\
\hline Type B & $6(7.1)$ & $2(4.2)$ & $4(11.1)$ & $4(9.5)$ & $2(4.8)$ \\
\hline Type G & $13(15.5)$ & $11(23)$ & $2(5.6)$ & $5(11.9)$ & $8(19)$ \\
\hline Unclassified & $1(1.2)$ & $0(0)$ & $1(2.8)$ & $1(2.4)$ & $0(0)$ \\
\hline Total & 84 & 48 & 36 & 42 & 42 \\
\hline
\end{tabular}

Types C, D, E and F were not observed in this study. 
Table II: The prevalence of sciatic nerve variation types with respect to symmetry

\begin{tabular}{|l|c|c|c|} 
& $\begin{array}{c}\text { Total } \\
\mathbf{n}(\%)\end{array}$ & $\begin{array}{c}\text { Males } \\
\mathbf{n}(\%)\end{array}$ & $\begin{array}{c}\text { Females } \\
\mathbf{n}(\%)\end{array}$ \\
\hline Bilateral (type A) & $28(66.7)$ & $15(35.7)$ & $13(31)$ \\
\hline Bilateral (variant types $\left.{ }^{\star}\right)$ & $3(7.1)$ & $2(4.8)$ & $1(2.4)$ \\
\hline $\begin{array}{l}\text { Unilateral (type A and variant } \\
\text { type) }\end{array}$ & $8(19)$ & $5(11.9)$ & $3(7.1)$ \\
\hline $\begin{array}{l}\text { Unilateral (variant types } \\
\text { ( })\end{array}$ & $3(7.1)$ & $2(4.8)$ & $1(2.4)$ \\
\hline Total & 42 & 24 & 18 \\
\hline
\end{tabular}

*Variant types included types B, G and the unclassified type

Table III: The region of sciatic nerve bifurcation with respect to sex and side

\begin{tabular}{|l|c|c|c|c|c|}
\hline & $\begin{array}{c}\text { Total } \\
\mathbf{n}(\%)\end{array}$ & $\begin{array}{c}\text { Male } \\
\mathbf{n}(\%)\end{array}$ & $\begin{array}{c}\text { Female } \\
\mathbf{n}(\%)\end{array}$ & $\begin{array}{c}\text { Right } \\
\mathbf{n}(\%)\end{array}$ & $\begin{array}{c}\text { Left } \\
\mathbf{n}(\%)\end{array}$ \\
\hline Thigh & $35(41.7)$ & $22(45.8)$ & $13(36.1)$ & $19(45.2)$ & $16(38.1)$ \\
\hline Gluteal & $25(29.8)$ & $18(37.5)$ & $7(19.4)$ & $11(26.2)$ & $14(33.3)$ \\
\hline Popliteal & $24(28.6)$ & $8(16.7)$ & $16(44.4)$ & $12(28.6)$ & $12(28.6)$ \\
\hline Total & 84 & 48 & 36 & 42 & 42 \\
\hline
\end{tabular}

bifurcated at the lower border of quadratus femoris, resulting in 33 limbs being measured. Of these, 17 were from the right side and 16 from the left; 20 were from males and 13 from females. The data were normally distributed ( $p>0.05)$. Bland-Altman and ANOVA tests revealed good inter- and intra-observer reliability $(p=0.99)$.

The average length of the nerve was $133.30 \mathrm{~mm} \pm 19.33 \mathrm{~mm}$, with no significant differences between side $(p=0.89)$ or sex $(p=0.90)$. The mean $\pm S D$ and range of the measurements are shown in Table IV. Independent t-tests revealed no significant differences between left and right $(p=0.89)$ or between males and females $(p=0.90)$.

\section{Discussion}

The SN was first described in detail by Ambroise Par'e in the 16th century, and in the 19th century, Cruveither observed variation in the bifurcation level. ${ }^{14}$ Variant types include the SN passing through the belly of the PM, or the high bifurcation of the SN into the TN and CFN in the gluteal region, with one of these branches possibly coursing through the PM. ${ }^{6,7}$ In this study, variation was observed in the position of the $\mathrm{SN}$ relative to the PM, the bifurcation level of the nerve, and the length of the nerve. Type A was the most common pattern in $76.2 \%$, which is lower than the pooled prevalence of $85.2 \%(95 \% \mathrm{Cl}: 78.4-87.0)$ reported by Tomaszewski et al. ${ }^{1}$ (Table $\mathrm{V}$ ). This suggests that variation in the relationship between the SN and PM may be more prevalent in South Africans. When compared with the prevalence of type A determined for other African samples (85.9\%), namely Ethiopian, Kenyan and Ugandan, variation was still more common in this study. ${ }^{1,4,12,15}$ Type $\mathrm{G}$ was the second most common pattern in $15.5 \%$, which is significantly higher than the pooled prevalence worldwide (0.6\%) and other African studies (3.8\%). ${ }^{1}$ Type B was observed in $7.1 \%$, which, although higher than the prevalence for other African studies (2.2\%), was similar to that reported for European studies (7.4\%). ${ }^{1}$ Types C, D, $\mathrm{E}$ and $\mathrm{F}$ although not present in our study, have been observed in other African studies with a prevalence of $2.6 \%, 0.4 \% 0.4 \%$ and $0.4 \%$ respectively. ${ }^{1}$ An unclassified type was observed unilaterally in one cadaver. Similar patterns have been described by Sabnis, where the TN was observed coursing beneath the inferior gemellus muscle. ${ }^{16}$

Although not significantly different, type B was more common in females $(11.1 \%)$ than in males $(4.2 \%)$, similar to the findings by Tomaszewski et al., who reported a prevalence of $20.1 \%$ and $11.7 \%$ respectively. ${ }^{1}$ These authors suggest that sex differences in the anatomy of the pelvic organs could predispose females to developing a type $B$ pattern. This in turn, may increase the risk of PS in females, although the link between variant anatomy and PS is controversial. ${ }^{17-20}$ Risk of iatrogenic SN injury during THA is, however, reported to be higher in females than in males, with suggested explanations related to the smaller size of the hip and associated muscles in females. ${ }^{21}$

Variations in the course of the SN have also been implicated in iatrogenic nerve injury during certain procedures, such as total hip arthroplasty and arthroscopy. ${ }^{11,22}$ The Moore/Southern and KocherLengenbeck approaches utilise the same inter-muscular planes. From a lateral incision, the gluteus maximus is split, in line with the muscle fibres, in the interval between the anterior third and the posterior two-thirds of the muscle, revealing the posterior edge of the greater trochanter and the short external rotators: piriformis, gemelli and obturator internus. The piriformis and the rest of the external rotators are then tagged with a suture, for re-attachment later, and cut $1.5 \mathrm{~cm}$ from the insertion on the greater trochanter. Retracting the external rotators posteriorly away from the trochanter effectively creates a sling that also pulls the SN out of the operating field..$^{8,9}$ The incidence of SN palsy following THA ranges from $0.05 \%$

Table IV: Length of the sciatic nerve

\begin{tabular}{|l|c|c|c|c|c|}
\hline & Total $(\mathrm{n}=\mathbf{3 3})$ & Males $(\mathrm{n}=\mathbf{2 0})$ & Females $(\mathrm{n}=\mathbf{1 3})$ & Right $(\mathrm{n}=\mathbf{1 7})$ & Left $(\mathrm{n}=\mathbf{1 6})$ \\
\hline Mean \pm SD $(\mathrm{mm})$ & $131.0 \pm 54.4$ & $137.1 \pm 64.1$ & $121.7 \pm 35.1$ & $132.4 \pm 46.4$ & $129.6 \pm 63.4$ \\
\hline Range $(\mathrm{mm})$ & $7-250$ & $7-250$ & $60-194$ & $34-240$ & $7-250$ \\
\hline
\end{tabular}

Table V: Comparison of the prevalence of pattern types observed in the present study with those reported by Tomaszewski et al. in a meta-analysis published in $2016^{1}$

\begin{tabular}{|c|c|c|c|c|c|c|c|}
\hline Sample & $\begin{array}{c}\text { Type A (\%) } \\
(95 \% \mathrm{CI})\end{array}$ & $\begin{array}{c}\text { Type B (\%) } \\
(95 \% \text { CI) }\end{array}$ & $\begin{array}{c}\text { Type C (\%) } \\
(95 \% \text { CI) }\end{array}$ & $\begin{array}{c}\text { Type D (\%) } \\
(95 \% \mathrm{CI})\end{array}$ & $\begin{array}{c}\text { Type E (\%) } \\
(95 \% \mathrm{CI})\end{array}$ & $\begin{array}{c}\text { Type F (\%) } \\
(95 \% \mathrm{Cl})\end{array}$ & $\begin{array}{c}\text { Type G (\%) } \\
(95 \% \text { CI) }\end{array}$ \\
\hline Present study & $76.2(66.4-84.0)$ & $7.1(2.9-12.9)$ & $0(0.0-0.0)$ & $0(0.0-0.0)$ & $0(0.0-0.0)$ & $0(0.0-0.0)$ & $15.5(9.4-24.7)$ \\
\hline Total & $85.2(78.4-87.0)$ & $9.8(6.5-13.2)$ & $1.9(0.6-3.8)$ & $0.8(0.0-2.2)$ & $0.5(0.0-1.5)$ & $0.4(0.0-1.4)$ & $0.6(0.0-1.9)$ \\
\hline Africa & $85.9(78.4-87.0)$ & $2.2(0.0-16.5)$ & $2.6(0.0-17.7)$ & $0.4(0.0-9.7)$ & $0.4(0.0-9.7)$ & $0.4(0.0-9.7)$ & $3.8(0.0-20.8)$ \\
\hline Europe & $87.7(81.0-91.2)$ & $7.4(3.8-11.6)$ & $2.0(0.4-4.7)$ & $0.9(0.0-3.0)$ & $0.3(0.0-1.4)$ & $0.2(0.0-1.2)$ & $1.1(0.0-3.3)$ \\
\hline Asia & $77.2(69.0-82.9)$ & $17.0(11.1-23.4)$ & $2.2(0.3-5.4)$ & $1.2(0.0-3.3)$ & $0.9(0.0-2.8)$ & $0.6(0.0-2.1)$ & $0.4(0.0-1.7)$ \\
\hline North America & 92.6 (77.9-99.4) & $2.8(0.0-10.4)$ & $0.6(0.0-5.0)$ & $0.3(0.0-4.1)$ & $0.2(0.0-3.5)$ & $0.2(0.0-3.5)$ & $0.2(0.0-3.5)$ \\
\hline South America & $85.0(76.4-92.8)$ & $9.8(3.9-18.0)$ & $1.4(0.0-4.8)$ & $0.8(0.0-3.6)$ & $0.5(0.0-2.8)$ & $1.4(0.0-4.8)$ & $0.5(0.0-2.8)$ \\
\hline
\end{tabular}


to $1.9 \% .^{21}$ During THA, the disruption of the insertion of the PM may predispose the $\mathrm{SN}$ to stretching, particularly in cases where a variant relationship exists. ${ }^{17}$ In addition, THA has been associated with limb lengthening, which places the SN under tension. ${ }^{23,24}$ In arthroscopy of the hip, certain variant positions result in the SN being situated closer to the placement of the posterolateral portal than the usual $\pm 3 \mathrm{~cm}$, increasing the risk of iatrogenic injury. ${ }^{10,11}$ Awareness of the position of the $\mathrm{SN}$ and careful positioning of the lower limb during procedures have been shown to decrease the incidence of iatrogenic injury by up to $50 \% .^{25}$

Piriformis syndrome (PS), although difficult to diagnose, is characterised by the following main symptoms: buttock pain that is aggravated during sitting, and external tenderness near the greater sciatic notch. ${ }^{26}$ In patients presenting with lower back or leg pain, the incidence of PS is around $5-6 \%{ }^{27}$ It has been suggested that variation in the relationship between the $\mathrm{SN}$ and $\mathrm{PM}$ is associated with PS. However, there is some debate as to the extent of this association, as not all individuals with PS have variant anatomy and vice versa. ${ }^{17-20}$ Type $B$ and other variants in which the SN, CFN or TN pass through the $\mathrm{PM}$, causing possible nerve impingement, have been linked with PS. Inflammation of the PM as a consequence of trauma may also result in PS, as inflammatory mediators released by the muscle irritate the $\mathrm{SN} .{ }^{27}$

Variation in the bifurcation point of the $\mathrm{SN}$ may result in the decreased effectiveness of popliteal blocks, as both the TN and CFN may not be anaesthetised if the nerve has divided in the gluteal region or superior thigh. ${ }^{4}$ In this study, variation was observed in the SN bifurcation level, with division in the thigh in $41.7 \%$, followed by the gluteal and popliteal regions in $29.8 \%$ and $28.6 \%$, respectively. This contradicts Ogeng'o et al.'s findings, in which the SN divided in the gluteal region in $2.4 \%$, in the thigh in $10.4 \%$, while $67.1 \%$ divided in the popliteal fossa, in a total of 82 cadavers. ${ }^{4}$ Interestingly, in $20.1 \%$ of cases, the nerve divided proximal to the PM. Kukiriza et al. reported variation in the bifurcation level in a Ugandan study, with no association between variation and the height of individuals. ${ }^{15}$ Patients considered for popliteal blocks should have the SN bifurcation point visualised with ultrasound prior to the procedure to ensure adequate anaesthesia of the leg and foot.

The length of the SN has been reported with little consistency with regard to any reference points. In this study, the inferior border of quadratus femoris was used, while other studies have measured the distance from the popliteal fossa to the bifurcation point (mean $65.43 \mathrm{~mm}$ ). ${ }^{1}$ Tomaszewski et al. proposed the distance from the transverse subcondylar plane be used, as it is a constant landmark. ${ }^{1}$ Depending on the formation of the SN and its bifurcation level, the length of the nerve is highly variable between individuals, with a range of $7-250 \mathrm{~mm}(133.30 \mathrm{~mm} \pm 19.33 \mathrm{~mm})$ recorded in our study.

There are limitations to this study. The length of the SN was measured from the inferior border of quadratus femoris, thus not reflecting the full length of the nerve from its formation in the pelvis. No biometric data (height or weight) was available for the cadavers, and limb length was not able to be measured, as medical students had already dissected the soft tissue structures. Thus, we were not able to consider the effect of height or limb length on the length of the nerve. There was no medical history available for the cadavers, and it was not known whether any of the individuals suffered from PS during their lifetimes. Therefore, we were unable to determine whether any of the variant types were associated with PS in our study. Future morphometric studies of the SN should take the height, weight and body mass index of the sample into account.

\section{Conclusion}

Variation in the relationship between the sciatic nerve and piriformis muscle was observed, namely types $A, B, G$ and a rare, unclassified pattern. Type B, possibly associated with piriformis syndrome, was more common in females than in males, while no differences were observed between left and right sides. The bifurcation level of the SN was variable, which may have implications for successful popliteal blocks. Bifurcation in the gluteal region was present in almost one-third of the sample. Awareness of the position of the nerve during surgical procedures around the hip and the variability that has been described may reduce the risk of iatrogenic injury. As the sciatic nerve is highly variable in its course and bifurcation, ultrasound should be used to identify the position of the nerve and its bifurcation point prior to nerve blocks. The use of ultrasound may increase the success rate and reduce complications associated with sciatic or popliteal blocks.

\section{Ethics statement}

All procedures were in accordance with the ethical standards of the responsible committee on human experimentation (institutional and national) and with the Helsinki Declaration of 1975 , as revised in 2008.

Written consent was obtained from the body donors, while consent for indigent individuals was obtained by the Department of Health, South Africa.

The body donation programme at our institution complies with the International Federation of Associations of Anatomists (IFAA)'s 'Recommendations of good practice for the donation and study of human bodies and tissues for anatomical examination'. In accordance with the policies of the institution's review board, it was not necessary to seek ethical approval as written informed consent was obtained from body donors for teaching and research purposes. Consent for the use of unclaimed bodies in this study was granted by the country's government health department.

\section{Declaration}

The authors declare authorship of this article and that they have followed sound scientific research practice. This research is original and does not transgress plagiarism policies.

\section{Author contributions}

TA contributed to the acquisition, analysis and interpretation of data for the work; drafting of the work; and final approval of the version to be submitted to the journal. GG contributed to the conception and design of the work, revising it critically for important intellectual content; and final approval of the version to be published.

RV contributed to revising the work critically for important intellectual content; and final approval of the version to be published.

$\mathrm{BH}$ contributed to revising the work critically for important intellectual content; and final approval of the version to be published.

$\mathrm{KK}$ contributed to the conception and design of the work; drafting the work, revising it critically for important intellectual content; and final approval of the version to be published.

\section{ORCID}

Asmall T (D) http://orcid.org/0000-0002-6839-6836 Gunston G (D) http://orcid.org/0000-0002-0780-0364 Venter R (iD http://orcid.org/0000-0003-0022-6969 Henry BM (iD http://orcid.org/0000-0002-8047-338X Keet K (D) http://orcid.org/0000-0003-3513-9232

\section{References}

1. Tomaszewski KA, Graves MJ, Henry BM, Popieluszko P, Roy J, Pekala PA, Hsieh WC, Vikse J, Walocha JA. Surgical anatomy of the sciatic nerve: a meta-analysis. J Orthop Res. 2016;34(10):1820-27. doi: 10.1002/jor.23186.

2. Shewale AD, Karambelkar RR, Umarji BN. Study of variations in the division, course and termination of the sciatic nerve. Journal of Krishna Institute of Medical Sciences University (JKIMSU). 2013;2(1):62-68.

3. Standring S. Gray's Anatomy: The Anatomical Basis of Clinical Practice. 41st edition. Elsevier: Amsterdam; 2016: pp 444-45, 451-53, 456, 781-85

4. Ogeng'o JA, El-Busaidy H, Mwika PM, Khanbhai MM, Munguti J. Variant anatomy of sciatic nerve in a black Kenyan population. Folia Morphologica. 2011;70(3):175-79.

5. Debbarma T, Debbarma K. A fetal study of sciatic nerve division and its clinical implications. Journal of Dental and Medical Sciences. 2016;15(2):27-29.doi: 10.9790/0853-15232729. 
6. Beaton LE, Anson BJ. The relation of the sciatic nerve and of its subdivisions to the piriformis muscle. Anat Rec. 1937;70:1-5. doi: 10.1002/ar.1090700102.

7. Smoll NR. Variations of the piriformis and sciatic nerve with clinical consequences: A review. Clin Anat. 2010;23:8-17. doi: 10.1002/ ca.20893.

8. Moretti VM, Post ZD. Surgical approaches for total hip arthroplasty. Indian J Orthop. 2017;51(4):368-76. doi: 10.4103/ ortho.IJOrtho31716.

9. Tosounidis TH, Giannoudis VP, Kanakaris NK, Giannoudis PV. The Kocher-Langenbeck approach. JBJS Essential Surgical Techniques. 2008;8(2):e18(1-7). doi: 10.2106/JBJS.ST.16.00102.

10. Alwattar BJ, Bharam S. Hip arthroscopy portals. Oper Tech Sports Med. 2011;19:74-80. doi: 10.1053/j.otsm.2010.12.003.

11. Byrd JWT. 2013. Operative Hip Arthroscopy. Third edition. Springer New York Heidelberg Dordrecht London. pp 102-103.

12. Desalegn M, Tesfay A. Variations of sciatic nerve its exit in relation to piriformis muscle in Northern Ethiopia. Int $J$ Pharm Sci. 2014;5(12) 953-56.

13. Anbumani TL, Thamarai SA, Anthony AS. Sciatic nerve and its variations: an anatomical study. IJAR. 2015;3(2):1121-27. doi: 10.16965/ijar.2015.175

14. Shrivastava T, Garg L, Mishra BK, Chhabra N. High division of sciatic nerve. Int J Res Med Sci. 2014;2(2):686-88.

15. Kukiriza J, Kiryowa H, Turyabahika J, Ochieng J, Ibingira CBR Levels of bifurcation of the sciatic nerve among Ugandans at School of Biomedical Sciences Makerere and Mulago Hospital Uganda. East Cent African J Surg. 2010;15:69-75.

16. Sabnis AS. Anatomical variations of sciatic nerve bifurcation in human cadavers. J Clin Res Lett. 2012;3(2):46-48.

17. Cassidy L, Walters A, Bubb K, Shoja MM, Tubbs RS, Loukas M. Piriformis syndrome: implications of anatomical variations, diagnostic techniques, and treatment options. Surg Radiol Anat. 2012;34:479-86. doi: 10.1007/s00276-012-0940-0.

18. Pecina M. Contribution to the etiological explanation of the piriformis syndrome. Acta Anaesthesiologica. 1979;105(2):181-87.

19. Vandertop WP, Bosma NJ. The piriformis syndrome: a case report. J Bone Joint Surg. 1991;73-A(7):1095-97.

20. Windisch G, Braun EM, Anderhuber F. Piriformis muscle: clinical anatomy and consideration of the piriformis syndrome. Surg Radiol Anat. 2007;29:37-45. doi:10.1007/s00276-006-0169-x.

21. Brown GD, Swanson EA, Nercessian OA. Neurologic injuries after total hip arthroplasty. Am J Orthop. 2008;37(4):191-97.

22. Pokorny D, Jahoda D, Veigl D, Pinskerova V, Sosna A. Topograhic variations of the relationship of the sciatic nerve and the piriformis muscle and its relevance to palsy after total hip arthroplasty. Surg Radiol Anat. 2006;28:88-91. doi: 10.1007/s00276-005-0056-x.

23. Edwards BN, Tullos HS, Noble PC. Contributory factors and etiology of sciatic nerve palsy in total hip arthroplasty. Clin Orthop. 1987;218:136-41.

24. Eggli S, Hankemayer S, Muller ME. Nerve palsy after leg lengthening in total replacement arthroplasty for developmental dysplasia of the hip. J Bone Joint Surg Br. 1999;81(5):843-45.

25. Navarro RA, Schmalzried TP, Amstutz HC, Dorey FJ. Surgical approach and nerve palsy in total hip arthroplasty. J Arthroplasty. 1995;10(1):1-5.

26. Hopayian K, Danielyan A. Four symptoms define the piriformis syndrome: an updated systematic review of its clinical features. Eur J Orthop Surg Traumatol. 2018;28:155-64. doi: 10.1007/ s00590-017-2031-8.

27. Benzon HT, Katz JA, Benzon HA, lqbal MS. Piriformis syndrome: Anatomic considerations, a new injection technique, and a review of the literature. Anesthesiology. 2003;98:1442-48. 